\title{
Alles eine Frage der Dosis?
}

$\mathrm{S}$ eit nahezu 100 Jahren stellt die spezifische subkutane Immuntherapie (SCIT) den Goldstandard zur kausal orientierten Behandlung von IgE-abhängigen Typ-I-Reaktionen dar. Jede Immuntherapie einschließlich neuer alternativer Formen zur klassischen Immuntherapie muss vor allem unter den beiden Aspekten Wirksamkeit und Sicherheit beurteilt werden $[8,9$, 17]. Deshalb wurden zahlreiche lokale Applikationsformen entwickelt und untersucht, da man sich hiervon ein besseres Nutzen-Risiko-Profil versprach. Die orale, bronchiale und nasale Immuntherapie konnten nicht überzeugen [2, 5].

Viele Daten, die in mehreren kontrollierten Studien belegt und in mehreren Meta-Analysen und Positionspapieren allergologischer Fachgesellschaften ausgewertet wurden, zeigen sowohl Wirksamkeit als auch Sicherheit der sublingualen Immuntherapie (SLIT) bei der allergischen Rhinitis [2, 3, 20] und lassen sie als eine viel versprechende Alternative erscheinen, zumal vielfältige immunologische Untersuchungen nahe legen, dass gerade die sublinguale Applikationsform eine Toleranzinduktion begünstigt. Erste Studien deuten zumindest darauf hin, dass keine wesentlichen Unterschiede in der Wirksamkeit der SLIT im Vergleich zur SCIT bestehen, wenngleich weitere Studien wünschenswert bleiben und auch geplant bzw. in der Durchführung sind. Gleichzeitig wurden bei der SCIT mehrere schwere systemische Reaktionen beobachtet, während bei der SLIT nur leichte bis moderate Reaktionen überwiegend lokaler Natur im Sinne eines oralen Allergiesyndroms auftraten [6]. Erste Hinweise zu Präventiveffekten liegen ebenfalls vor. So scheint eine 5-jährige sublinguale Behandlung bei allergischem Asthma einen anhaltenden Effekt über 4 bis 5 Jahre zu haben [18]. Möglicherweise reduziert die SLIT auch die Zahl der Pati-

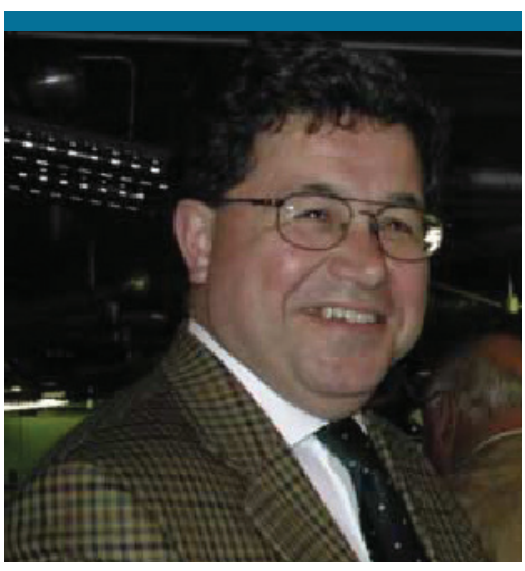

Prof. Dr. Hans F. Merk, Klinik für Dermatologie und Allergologie, Universitätsklinikum der Rheinisch-Westfälischen Technischen Hochschule, Aachen

enten mit neu manifestiertem allergischen Asthma [16].

Nach wie vor gibt es neben eindeutig positiven Studien zur SLIT auch solche mit zweifelhaften oder negativen Ergebnissen [12]. Bei einigen nicht eindeutigen Studien mögen hierfür eine zu geringe natürliche Allergenexposition in der Observationsperiode, vorbestehende Unterschiede zwischen Verum- und Kontrollgruppe, zusätzliche begleitende symptomatische Maß„Viele Daten zeigen sowohl nahmen oder der Einsatz Wirksamkeit als auch der heute als nicht wirkSicherheit der sublingualen sam eingestuften SubImmuntherapie bei der lingual-Spit-Methode allergischen Rhinitis und verantwortlich sein. Ein lassen sie als eine viel weiterer Grund könnte versprechende Alternative aber auch in der verwenerscheinen." deten Dosierung liegen. Diese Vermutung wird auch in einer ganz aktuellen Meta-Analyse zur SLIT wieder geäußert [19, 20]. Es mehren sich auf der anderen Seite die Hinweise, dass die hoch dosierte SLIT der niedrig dosierten SLIT überlegen sein könnte. So ist die klinische Effektivität nach den von Malling definierten Kriterien [11] bei Studien mit einer hohen kumulativen Dosis eher moderat bis hoch, während bei niedriger Dosis häufiger keine oder nur eine geringe Wirksamkeit zu beobachten ist [1]. Diese Be- obachtungen bestätigen, dass auch immunologischePhänomenedosisabhängig sind. Der IgE-Anstieg während saisonaler Allergenexposition kann durch eine hohe sublinguale Dosis vermieden werden, jedoch nicht durch eine niedrige Dosis [13].

Weitere Daten unterstützen das theoretische Konzept der SLIT, die sich den wichtigen physiologischen Mechanismus der oralen Toleranz zu Nutze macht. Dabei erleichtern Hochdosisschemata offenbar das Aufnehmen ausreichender Mengen von Allergenen durch dendritische Zellen in der Mundschleimhaut [15]. Auch sind hohe Antigendosen sinnvoll, um einen starken initialen Impuls der T-LymphozytenStimulation zu erreichen [14], der zum Entstehen des T-Zell-vermittelten Immungedächtnisses und zur Langzeittoleranz führt. Auch ist nach diesen Daten die Wirkung der SLIT nicht lokal begrenzt. Die lokale Verabreichung des Allergens während der Hyposensibilisierung mit der Folge der Zirkulation der Allergen-spezifischen effektorischen T-Zellen durch den Organismus und das Fortbestehen der Gedächtniszellen führt zu einer sowohl systemisch als auch mukosal schützenden Immunantwort. Auch die gute Verträglichkeit der SLIT ist durch die lokale Allergenapplikation zu erklären. In der Mundschleimhaut ist nach diesen Daten im Vergleich zur Haut nur eine begrenzte Anzahl von pro-inflammatorischen Zellen wie zum Beispiel Mastzellen vorhanden.

Wenn auch der immunologische Mechanismus der SLIT noch nicht vollständig aufgeklärt ist, scheinen diese Konzepte konsistent mit Studien zur Wirksamkeit. So ergeben sich in der randomisierten kontrollierten Studie von André et al. (S. S6) deutliche Hinweise, dass die verwendete Allergendosis auch klinisch relevant zu sein scheint. Es handelt sich bei dieser Studie zwar nicht um eine klassische Dosisfindungsstudie, aber die hier durchgeführte Subgruppenanalyse legt nahe, dass eine höhere Einzeldosis ein größeres Ausmaß an 
Wirksamkeit zur Folge hat. So wurde bei den Patienten mit der niedrigen Dosierung bei den Symptom- und Medikationsscores kein signifikanter Unterschied im Vergleich zu Plazebo beobachtet, während sich signifikante Verbesserungen der Symptome bei den Patienten mit der hohen Dosierung zeigten.

Die Meta-Analyse aus der Arbeitsgruppe von G. Ciprandi (S. S14) legt nahe, dass im Gegensatz zur Wirksamkeit bei der Verträglichkeit keine strenge Dosisabhängigkeit besteht. Die kumulative Dosis der 25 ausgewerteten Studien zur SLIT lag zwischen dem 1- und 500 fachen der üblichen Dosis bei der SCIT. Die
„Es gibt zahlreiche Anhaltspunkte dafür, dass die Dosis sowohl für die immunologische als auch die klinische Wirkung eine Rolle spielt."

träglichkeit der SLIT bei der allergischen Rhinitis. Es gibt zahlreiche Anhaltspunkte dafür, dass die Dosis sowohl für die immunologische als auch die klinische Wirkung eine Rolle spielt.

Nichtsdestoweniger darf die SLIT heute als eine ernsthafte Erweiterung des therapeutischen Spektrums bei der allergischen Rhinitis gelten. Die empirisch gewonnene ARIA-Empfehlung der mindestens 50- bis 100fachen kumulativen Sublingualdosis im Verhältnis zur subkutanen Gabe stellt nach wie vor eine pragmatische Lösung zur Einschätzung der zu erwartenden Wirksamkeit dar. Man darf gespannt und niedriger Dosis wurde gemäß der ARIA-Empfehlung [2] beim 50 fachen der subkutanen Dosis gezogen. Es ergaben sich Raten zwischen 27,6 und 49 unerwünschten Ereignissen auf 10.000 Einnahmen, wobei speziell bei den lokalen und gastrointestinalen Reaktionen diese Rate unter niedriger Dosis höher war als unter hoher Dosis.

Wenngleich sich somit die Evidenz für die bessere Effektivität einer hohen Dosis zunehmend verdichtet, steht der praktisch tätige Allergologe im klinischen Alltag vor der Herausforderung, die Dosierung der zur Verfügung stehenden Sublingualpräparate richtig einzuschätzen. Da die Hersteller von Allergenextrakten der Forderung einer einheitlichen Standardisierung bis heute noch nicht nachgekommen sind [10], wird im Beitrag von R. Mösges (S. S22) die biologische Aktivität verschiedener Extrakte in Relation zueinander gesetzt. Hier zeigen sich erhebliche Unterschiede um den Faktor acht bis 22 in der wöchentlich applizierten Dosis. Diese Untersuchung kann zwar eine Dosisfindungsstudie oder Vergleichsstudie mit klinischen Parametern nicht ersetzen, mag aber als Hilfestellung zur Einschätzung der biologischen Potenz eines Sublingualextraktes dienen.

Die Forderung nach eindeutigen Dosisfindungsstudien [4,7] ist nach wie vor berechtigt. Die heute zur Verfügung stehenden Daten belegen aber eindeutig die Wirksamkeit und Ver- sein, wann Dosisfindungsstudien diese erste Evidenz endgültig bestätigen und auch Aufschluss über das optimale Applikationsschema geben.

\section{Literatur}

1. André C. The development of local immunotherapy. Recent Res Devel Allergy Clin

2. Bousquet J, van Cauwenberge P, Khaltaev N. Allergic rhinitis and its impact on asthma. J

3. Bousquet J, Lockey R, Malling HJ. Allergen immunotherapy: therapeutic vaccines for allergic diseases. A WHO position paper. Allergy 1998; 53 Suppl 44: 1-42

4. Calderon M, Rak S, Durham SR. Grass pollen tablets for sublingual immunotherapy in seasonal allergic rhinitis. J

5. Kägi MK, Wüthrich B. Different methods of local allergen-specific immunotherapy. Allergy 2002; 57: 379-88

6. Khinchi MS, Poulsen LK, Carat F, André C, Hansen AB, Malling HJ. Clinical efficacy of sublingual and subcutaneous birch pollen allergen-specific immunotherapy: a randomized, placebo-controlled, doubleblind, double-dummy study. Allergy 2004; 59: 45-53

7. Kleine-Tebbe J, Bergmann K-C, Bufe A, Friedrichs F, Fuchs T, Hirsch T, Klimek L, Lepp U, Przybilla B, Rakoski J, Rebien W, Saloga J, Schultze-Werninghaus G, Virchow J-C. Aktueller Stellenwert der sublingualen Immuntherapie bei allergischen Krankheiten. Allergologie 2004; 27: 381-84

8. Lockey RF, Benedict LM, Turkeltaub PC, Bukantz SC. Fatalities from immunotherapy (IT) and skin testing (ST). J Allergy Clin Immunol 1987; 79: 660-77

9. Lüderitz-Püchel U, Keller-Stanislawski B, Haustein D. Neubewertung des Risikos von Test- und Therapieallergenen. BundesImmunol 2001; 2: 45-53 Allergy Clin Immunol 2001; 108: S147-334 Allergy Clinical Immunol, 2005; 115: S65. gesundheitsblatt Gesundheitsforschung Gesundheitsschutz 2001; 44: 709-18

10. Maetzke J, Hinrichs R, Scharffetter-Kochanek K. Notwendigkeit der standardisierten Qualität der sublingualen Immuntherapie. Allergo J 2005; 14: 253-4

11. Malling HJ. Immunotherapy as an effective tool in allergy treatment. Allergy 1998; 53: 461-72

12. Malling $\mathrm{HJ}$. Is sublingual immunotherapy clinically effective? Curr Opin Allergy Clin Immunol 2002; 2: 523-31

13. Marcucci F, Sensi L, Di Cara G, Incorvaia C, Frati F. Dose dependence of immunological response to sublingual immunotherapy. Allergy 2005; 60: 952-6

14. Martin-Fontecha A, Sebastiani S, Hopken UE, Uguccioni M, Lipp M, Lanzavecchia A, Sallusto F. Regulation of dendritic cell migration to the draining lymph node: impact on T lymphocyte traffic and priming. J Exp Med 2003; 198: 615-21

15. Noirey N, Rougier N, André C, Schmitt D, Vincent $C$. Langerhans-like dendritic cells generated from cord blood progenitors internalize pollen allergens by macropinocytosis, and part of the molecules are processed and can activate autologous naive T lymphocytes. J Allergy Clin Immunol 2000; 105: 1194-201

16. Novembre E, Galli E, Landi F, Caffarelli C, Pifferi M, De Marco E, Burastero SE, Calori G, Benetti L, Bonazza P, Puccinelli P, Parmiani S, Bernardini R, Vierucci A. Coseasonal sublingual immunotherapy reduces the development of asthma in children with allergic rhinoconjunctivitis. J Allergy Clin Immunol 2004; 114: 851-7

17. Reid MJ, Lockey RF, Turkeltaub PC, Platts-Mills TA. Survey of fatalities from skin testing and immunotherapy 1985-1989. J Allergy Clin Immunol 1993; 92: 6-15

18. Di Rienzo V, Marcucci F, Puccinelli P, Parmiani S, Frati F, Sensi L, Canonica GW, Passalacqua G. Long-lasting effect of sublingual immunotherapy in children with asthma due to house dust mite: a 10-year prospective study. Clin Exp Allergy 2003; 33: 206-10

19. Wilson DR, Torres Lima M, Durham SR. Sublingual immunotherapy for allergic rhinitis. Cochrane Database Syst Rev 2003: CDoo2893

20. Wilson DR, Torres Lima M, Durham SR. Sublingual immunotherapy for allergic rhinitis: systematic review and metaanalysis. Allergy 2005; 60: 4-12 